\title{
Marcadores para una herramienta de evaluación de Wikis
}

\section{Pilar I. Vidal-Carreras ${ }^{a}$, Lourdes Canos-Daros ${ }^{b}$ y Jose P. Garcia-Sabater}

${ }^{a}$ ROGLE, Departamento de Organización de Empresas, Universidad Politécnica de Valencia, pivicar@omp.upv.es, ${ }^{b}$ ROGLE, Departamento de Organización de Empresas, Universidad Politécnica de Valencia, loucada@omp.upv.es y ${ }^{\mathrm{c}}$ ROGLE, Departamento de Organización de Empresas, Universidad Politécnica de Valencia, jpgarcia@omp.upv.es

\begin{abstract}
A wiki is a website that can be edited easily by a user or any number of users. It can be stated that its use in the field of education is reasonably extended. However, assesment of the wiki is an emerging research line. According to the Spanish higher education system, raise the wiki activity as evaluable activity involves the evaluation by the teacher of both the quality of the wiki itself as competences developed through the activity. The electronic support of the wiki facilitates obtaining several automatic indicators such as number of words, number of links, number of figures, number of sources, etc., that could facilitate the task of the evaluation. Establishing connections between these indicators and the quality of the wiki as well as the competences developed during the activity is the main objective of this work.
\end{abstract}

Keywords: wiki, collaborative work, assesment, competences

\begin{abstract}
Resumen
Una wiki es un sitio web que puede ser editado fácilmente por cualquier número de usuarios. Se puede afirmar que su uso en el ámbito de la educación está razonadamente extendido. Sin embargo, la evaluación de la wiki es una línea de trabajo emergente. En el marco del sistema universitario español, plantear la actividad wiki como actividad evaluable implica la evaluación por parte del profesor tanto de la calidad de la propia wiki como de la/s competencia/s desarrolladas a través de la actividad. El soporte electrónico de la wiki facilita la obtención de una serie de marcadores automáticos tales como $n^{o}$ de palabras, $n^{o}$ de links, $n^{o}$ de figuras, $n^{o}$ de fuentes, etc., que podrían facilitar la tarea de evaluación del profesor. El establecimiento de las conexiones entre dichos marcadores y la calidad de la wiki así como las competencias desarrolladas durante la actividad es el objetivo fundamental del presente trabajo.
\end{abstract}

Palabras clave:wiki, trabajo colaborativo, evaluación, competencias

\section{Introducción}

En Wikipedia (Fundación Wikimedia) encontramos el ejemplo más popular y exitoso hasta el momento (Alexa) de la creación de una wiki, en este de caso de una enciclopedia on line, 
libre, políglota y editada colaborativamente. De acuerdo con Wikipedia, una wiki (del hawaiano wiki, 'rápido') es el nombre que recibe un sitio web cuyas páginas pueden ser editadas directamente desde el navegador, donde los usuarios crean, modifican o eliminan contenidos que, generalmente, comparten. Es un sistema de trabajo que utilizan los sitios web para editar o crear un contenido de forma rápida y sencilla. Siguiendo a Leuf y Cunningham (1999) un wiki es un conjunto de páginas web vinculadas, creado a través del desarrollo incremental por uno o un grupo de usuarios que colaboran así como el software utilizado para gestionar el conjunto de páginas web (Wagner, 2004).

El uso de la wiki se extiende a varios ámbitos para aprovechar sus ventajas inherentes. Esta son principalmente la facilidad y sencillez en la que es posible crear y mejorar las páginas de forma inmediata (Fundación Wikimedia), así como las asociadas al trabajo colaborativo como es permitir la interacción entre todos los miembros de la comunidad educativa, para un mayor y mejor seguimiento del proceso de enseñanza-aprendizaje. (Lamb, 2007; Pujolás, 2009). En el ámbito de la educación encontramos una revisión de su estado actual en los trabajos de Fountain (2005) y Parker y Chao (2007). Concretamente en la docencia universitaria existen varias experiencias del uso de wiki a señalar. Algunas de las más completas se refieren al campo de la informática, por ejemplo, WikiUNIX (aprendizaje de la administración de sistemas UNIX), Tutorial Wiki libSDL (apoyo al desarrollo de videojuegos en lenguaje $\mathrm{C}++$ con la biblioteca libre multimedia y multiplataforma libSDL), R-Wiki (acerca del paquete estadístico R) e IberOgre (desarrollo de videojuegos en 3D), entre otros. También existen experiencias relativas a la realización del trabajo fin de grado como LearningWx (Palomo-Duarte, 2012).

Al utilizarse la wiki como actividad docente en el ámbito universitario existe una línea de investigación importante relacionada con la evaluación de la misma. En este marco es en el que se sitúa el presente trabajo. Al analizar la literatura sobre evaluación de wikis encontramos trabajos que establecen marcadores automáticos a medir como pueden ser número de usuarios, número de bytes, número de páginas, ficheros subidos, visitas etc. Estos marcadores en ocasiones están integrados en una herramienta automática para realizarlo como StatMediaWiki y AssessMediaWiki (Palomo-Duarte, 2012; 2014), HistoryFlow (Viégas, 2004), WikiXRay (Ortega, 2007) o WattleTrees (Kay, 2006). Sin embargo encontramos un posible gap en esta área, que intentamos cubrir mediante este trabajo. Respecto a los marcadores, por una parte existen marcadores útiles que no están incluidos en ninguna de estas herramientas documentadas y, por otra, no existe una herramienta automática de medida que integre todos los marcadores requeridos tanto documentados como no, que permitan una completa evaluación de la wiki. Respecto a la utilidad de los marcadores documentados no siempre aparece una referencia clara a cómo poder utilizarlos para la evaluación de la wiki. Es una realidad que las enseñanzas universitarias están cambiando su focalización hacia un modelo basado en la adquisición de competencias, por lo que también resultaría muy interesante aprovechar estos marcadores para poder evaluar competencias en la wiki. De este modo, este artículo pretende como objetivo final plantear el listado completo de marcadores que debiera incluir cualquier herramienta destinada a facilitar la evaluación automática de una wiki, tanto desde el punto de vista de la evaluación de la propia wiki desarrollada como de las competencias 
adquiridas en el proceso. La estructura del artículo es la siguiente: en el apartado 2 se describen los objetivos, en el 3 se presenta el desarrollo del trabajo, en el 4 se presentan los resultados y en el 5 se presentan las conclusiones.

\section{Objetivos}

Los objetivos perseguidos con este trabajo son los siguientes:

- Determinar qué competencias pueden evaluarse a través de la wiki

- Identificar qué aspectos deben considerarse para poder evaluar una wiki

- Establecer una lista de marcadores/requerimientos que debiera contener una herramienta automática de medida en las wikis que incluyan todos los aspectos necesarios tanto para la evaluación de las competencias en la wiki como de la propia wiki

Cabe señalar que los dos primeros objetivos son requeridos a priori para la consecución del último objetivo que consiste en el resultado buscado con la innovación. De esta manera los dos primeros objetivos se incluirán en el apartado 3 de desarrollo de la innovación y el objetivo final se presentará en el apartado 4 de resultados.

\section{Desarrollo de la innovación}

La primera parte del trabajo analiza los aspectos relacionados con la evaluación de las competencias. La segunda parte considera cómo poder evaluar una actividad wiki propiamente dicha. La última parte lista los marcadores automáticos que pueden obtenerse de la wiki.

\subsection{Competencias a evaluar en la wiki}

Como se ha comentado, las enseñanzas universitarias están cambiando su focalización hacia un modelo basado en la adquisición de competencias. Estas competencias, sobre todo las de carácter transversal, servirán para que los alumnos aprendan a continuar su propia educación después de finalizar los estudios reglados, es decir, aprendan a lo largo de la vida (Mourtos, 2003), lo que les permitirá enriquecerse como personas y como profesionales. De este modo, el estudiante adquiere destrezas y habilidades instrumentales, interpersonales y sistémicas que son clave para el éxito. En este contexto, la evaluación del nivel alcanzado en las competencias y el seguimiento del aprendizaje son temas capitales para el desarrollo de las capacidades de cada estudiante. Para hacer un buen seguimiento hay que establecer controles y sistemas de asesoramiento para ofrecer orientaciones y ayuda ante una dificultad encontrada en el proceso, además de corregir los errores o desviaciones que puedan aparecer. Dicho seguimiento ha de ser cada vez menor, a medida que el estudiante va superando distintos cursos, pues el objetivo final es alcanzar cierta autonomía (Villa, 2007). La evaluación de las competencias debe estar detallada en la guía docente de cada asignatura y se debe presentar al comenzar la asignatura, explicando las competencias involucradas en la asignatura y las actividades que se realizarán para alcanzar el nivel definido para cada competencia (Alsina, 2013).

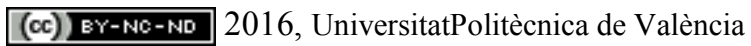

Congreso In-Red (2016) 
Con el diseño de la actividad de la wiki se pretende evaluar algunas competencias transversales. En la Universitat Politècnica de València, de acuerdo con el Plan Estratégico UPV2020, en el que el primer reto es "La Universitat Politècnica de València tiene como objetivo avanzar hacia modelos de formación que logren que sus estudiantes adquieran las competencias necesarias para poder tener una adecuada inserción laboral", se han definido 13 competencias transversales que están presentes en todos los planes de estudio de la Universidad, sea cual sea su especialidad (ver una breve definición de cada una de ellas en Tabla 1).

Tabla 1. Competencias transversales UPV.

\begin{tabular}{|c|c|}
\hline $\begin{array}{l}\text { CT-01. Comprensión e } \\
\text { integración }\end{array}$ & $\begin{array}{l}\text { Demostrar la comprensión en integración del conocimiento } \\
\text { tanto de la propia especialización como en otros contextos más } \\
\text { amplios }\end{array}$ \\
\hline $\begin{array}{l}\text { CT-02. Aplicación } \quad \mathrm{y} \\
\text { pensamiento práctico }\end{array}$ & $\begin{array}{l}\text { Aplicar los conocimientos a la práctica, atendiendo a la } \\
\text { información disponible, y estableciendo el proceso a seguir } \\
\text { para alcanzar los objetivos con eficacia y eficiencia }\end{array}$ \\
\hline $\begin{array}{l}\text { CT-03. Análisis } \quad \mathrm{y} \\
\text { resolución de problemas }\end{array}$ & $\begin{array}{l}\text { Analizar y resolver problemas de forma efectiva, identificando } \\
\text { y definiendo los elementos significativos que los constituyen }\end{array}$ \\
\hline $\begin{array}{l}\text { CT-04. Innovación, } \\
\text { creatividad } \\
\text { emprendimiento }\end{array}$ & $\begin{array}{l}\text { Innovar para responder satisfactoriamente, y de forma original, } \\
\text { a las necesidades y demandas personales, organizativas y } \\
\text { sociales, con el propósito de aportar valor, con una actitud } \\
\text { emprendedora }\end{array}$ \\
\hline $\begin{array}{lll}\text { CT-05. } & \text { Diseño } & \text { y } \\
\text { proyecto } & & \end{array}$ & $\begin{array}{l}\text { dirigir, desarrollar y evaluar una idea de manera eficaz } \\
\text { ncretarla en un servicio o producto }\end{array}$ \\
\hline $\begin{array}{lr}\text { CT-06. Trabajo } \\
\text { equipo y liderazgo }\end{array}$ & $\begin{array}{l}\text { Trabajar y liderar equipos de forma efectiva para la } \\
\text { consecución de objetivos comunes de un grupo de personas, } \\
\text { contribuyendo al desarrollo personal y profesional de los } \\
\text { mismos }\end{array}$ \\
\hline $\begin{array}{l}\text { CT-07. Responsabilidad } \\
\text { ética, medioambiental y } \\
\text { profesional }\end{array}$ & $\begin{array}{l}\text { Actuar con responsabilidad ética, medioambiental y profesional } \\
\text { ante uno mismo y los demás }\end{array}$ \\
\hline $\begin{array}{l}\text { CT-08. Comunicación } \\
\text { efectiva }\end{array}$ & $\begin{array}{l}\text { Comunicarse de manera efectiva, tanto de forma oral como } \\
\text { escrita, utilizando adecuadamente los recursos necesarios y } \\
\text { adaptándose a las características de la situación y de la } \\
\text { audiencia }\end{array}$ \\
\hline $\begin{array}{l}\text { CT-09. } \\
\text { crítico }\end{array}$ & $\begin{array}{l}\text { Desarrollar un pensamiento crítico que se interese por los } \\
\text { fundamentos en los que se asientan las ideas, acciones y juicios, } \\
\text { tanto propios como ajenos }\end{array}$ \\
\hline CT-10. Conocimiento de & Ic \\
\hline
\end{tabular}

(cc) EY-NC-ND 2016, UniversitatPolitècnica de València 


\begin{tabular}{|l|l|}
\hline $\begin{array}{l}\text { problemas } \\
\text { contemporáneos }\end{array}$ & $\begin{array}{l}\text { campo de especialización así como en otros campos del } \\
\text { conocimiento }\end{array}$ \\
\hline $\begin{array}{l}\text { CT-11. Aprendizaje } \\
\text { permanente }\end{array}$ & $\begin{array}{l}\text { Utilizar el aprendizaje de manera estratégica, autónoma y } \\
\text { flexible, a lo largo de toda la vida, en función del objetivo } \\
\text { perseguido }\end{array}$ \\
\hline $\begin{array}{l}\text { CT-12. Planificación y } \\
\text { gestión del tiempo }\end{array}$ & $\begin{array}{l}\text { Planificar adecuadamente el tiempo disponible y programar las } \\
\text { actividades necesarias para alcanzar los objetivos tanto } \\
\text { académico-profesionales como personales }\end{array}$ \\
\hline $\begin{array}{l}\text { CT-13. Instrumental } \\
\text { específica }\end{array}$ & $\begin{array}{l}\text { Utilizar adecuadamente las herramientas actualizadas } \\
\text { necesarias para la práctica de la profesión }\end{array}$ \\
\hline
\end{tabular}

Fuente: Elaboración propia a partir de documentos de trabajo ICE-UPV.

Consideramos diferentes elementos para definir cada rúbrica (ICE, 2015). El primero es el nivel de logro, siempre creciente (Universitat Politècnica de Catalunya, 2008). Según este criterio el estudiante ha de ser evaluado, al menos una vez, durante los dos primeros cursos del grado (nivel de dominio I), siendo este un aprendizaje dirigido en el que todo el trabajo está pautado; una segunda vez, en los puntos de control establecidos para cada título en tercer y cuarto curso de grado (nivel de dominio II), a través de un aprendizaje guiado que permita cierta autonomía en el estudiante; y durante la formación del máster (nivel de dominio III) en el que el aprendizaje es autónomo. En esta línea, los indicadores son otro elemento importante, definidos como concreciones del resultado de aprendizaje de cada nivel de dominio, junto con los descriptores, que definen el comportamiento del estudiante con respecto a cada indicador. Para la calificación se ha establecido una escala de cuatro valores según el nivel de desarrollo de cada estudiante en cada competencia:

- $\mathrm{A} \rightarrow$ excelente

- $\mathrm{B} \rightarrow$ adecuado

- $\mathrm{C} \rightarrow$ en desarrollo

- $\quad \mathrm{D} \rightarrow$ no alcanzado

En concreto, las competencias que se desarrollarán utilizando la wiki y los aspectos particulares que se considerarán para la evaluación son:

- CT-01 Comprensión e integración. Para incluir un contenido en el wiki es necesario leer lo que ya se encuentra en él, contribuyendo al proceso de reflexión. No se trata de hacer un copia-pega, sino de buscar información de diferentes fuentes y ser capaz de entenderla e integrarla en la wiki.

- CT-06Trabajo en equipo y liderazgo. La wiki tiene parte de trabajo individual pero al final se crea a través del trabajo del grupo.

- CT-08 Comunicación efectiva (vertiente escrita). El propio proceso de escritura va a desarrollar la competencia lingüística tan perjudicada actualmente.

- CT-07Responsabilidad ética, medioambiental y profesional. Sin realizar plagios y siempre citando las fuentes bibliográficas. Se fomenta que los alumnos compartan 
sus contenidos y experimenten como esto puede enriquecerles de muchas formas, fomentando la responsabilidad social.

- CT-11 Aprendizaje permanente. De acuerdo con Kolodner y Guzdian (1996) el aprendizaje colaborativo en entornos tecnológicos crea un conocimiento más profundo, que contribuye al aprendizaje permanente.

- CT-12Planificación y gestión del tiempo: Tanto si el trabajo se realiza de forma individual y como grupo, el estudiante debe organizar el tiempo disponible para realizar la actividad.

- CT-13 Instrumental específica. Los estudiantes aprender el uso de la herramienta wiki.

Somos conscientes de que la evaluación de todas estas competencias transversales en la misma asignatura es un hecho muy ambicioso, por lo que corresponderá a los profesores elegir la evaluación de la más adecuada de acuerdo con la coordinación global que se esté llevando a cabo en el título. Del mismo modo, hay que tener en cuenta si la asignatura en la que se desarrolla la wiki es punto de control de alguna de estas competencias o sólo se trabajan para reforzar su adquisición, aspecto que también depende de la coordinación general del título.

Por otra parte, cabe mencionar que existen otras experiencias en las que la wiki permite evaluar competencias diferentes, al menos en lo que a su nomenclatura se refiere, en función de la definición realizada por cada institución docente. Por ejemplo, Palomo-Duarte et al. (2012) valoran las siguientes competencias: adaptación al cambio, aprendizaje, innovación y trabajo en equipo.

\subsection{Evaluación de la propiawiki}

El profesor debe evaluar la actividad de una wiki de acuerdo con una nota que irá de acuerdo con el sistema actual de 0 a 10 . El objetivo es que los alumnos desarrollen a través de la wiki contenidos de calidad. Para la valoración de la calidad de la wiki se debe considerar respecto a las aportaciones realizadas por los alumnos:

- Profundidad

- Extensión

- Estructura

- Claridad en la lectura

- Conexión con conceptos

- Oportunidad

- Actualidad

Como se observa en la evaluación de las aportaciones a la wiki que son las que forman la propia wiki en sí misma, hay aspectos que también estén relacionados con las competencias. Por ejemplo, la claridad en la lectura de la aportación del alumno está directamente relacionada con la competencia de comunicación efectiva (escrita).

\subsection{Marcadores posibles de conteo en la wiki}

(c) ) BY-NC-ND 2016, UniversitatPolitècnica de València 
Al desarrollar el contenido del trabajo en un soporte wiki hay varios indicadores que pueden medirse automáticamente y que pueden contribuyen a la valoración de las competencias y de la calidad de la wiki. Estos son:

- Calidad ortográfica y gramatical del texto presentado. A través de los revisores automáticos de ortografía y gramática.

- $\mathrm{N}^{\mathrm{o}}$ de palabras. En su propia wiki o al revisar las wikis de otros.

- $\mathrm{N}^{\mathrm{o}}$ de párrafos. En su propia wiki o al revisar las wikis de otros.

- $\mathrm{N}^{\mathrm{o}}$ de frases. En su propia wiki o al revisar las wikis de otros.

- $\mathrm{N}^{\mathrm{o}}$ de figuras. En su propia wiki o al revisar las wikis de otros.

- $\mathrm{N}^{\mathrm{o}}$ de fuentes consultadas. En su propia wiki o en wikis de otros.

- $\mathrm{N}^{\mathrm{o}}$ de links externos. En su propia wiki o al revisar las wikis de otros.

- $\mathrm{N}^{\circ}$ de link internos. En su propia wiki o en wikis de otros.

- Conteo de actividad del alumno en la wiki de acuerdo con el tiempo. Para aunar la actividad del alumno es interesante utilizar la medida de bytes.

Hay que señalar que estos marcadores se han establecido suponiendo una actividad a realizar en la wiki tanto de manera individual como colectiva. Cada alumno o grupo de alumnos tendría asignado por parte del profesor qué actividad debía hacer en la wiki por lo que se podía asumir de algún modo la propiedad inicial de cada alumno o grupo de alumnos, que luego era completada de manera colaborativa. Por ejemplo: cada grupo de alumnos debe documentar los resultados de una práctica. Existen $\mathrm{X}$ prácticas en la asignatura, cada grupo de alumnos documenta una (que se considera la wiki propia), y luego todos colaboran para mejorar el resultado global del conjunto. Cuando hablamos de wiki, puede considerarse tanto que cada alumno haga una wiki diferente, o que cada una sea una página de la misma wiki.

\section{Resultados}

En ese apartado se plantea la relación que puede existir entre en el listado de marcadores y los aspectos y competencias a evaluar en el wiki. La tabla 2 muestra dicha relación.

Como puede observarse la profundidad en las aportaciones wiki incluye marcadores propios de la extensión. Dos ideas profundas cada una en un párrafo profundo es más que una sola idea profunda en un párrafo profundo. La claridad en la lectura se relaciona con la ortografía y la gramática al igual que la CT-08 Comunicación efectiva como se ha comentado anteriormente. La conexión con conceptos se puede valorar tanto a través de los enlaces internos como externos, y las fuentes consultadas. La actualidad del contenido se refleja en las fuentes consultadas así como los links externos. La CT-07 Responsabilidad ética se muestra con las fuentes citadas. La CT-12 Planificación y gestión del tiempo se muestra a través del conteo de la actividad de los alumnos en el tiempo. Es claro que al ser capaces de escribir palabras, insertar imágenes, fuentes, links demuestra el aprendizaje de la wiki como software (CT-13 Instrumental específica). La CT-11 Aprendizaje permanente se consigue con la mejora autónoma del alumno en sus conocimientos, al añadir aportaciones

(cc) EY-NC-ND 2016, UniversitatPolitècnica de València

Congreso In-Red (2016) 
en su propia wiki o en la de otros. Al tratarse de una evolución debe considerar el conteo de la actividad en el tiempo. No es sencillo la medición de a capacidad de CT-06 Trabajo en equipo y liderazgo a través de los marcadores, pero en el caso de que la actividad se deba hacer entre un grupo de alumnos la distribución de la actividad de los mismo en el tiempo puede dar alguna pista sobre cómo se ha desarrollado la competencia. Tanto la oportunidad del contenido como la estructura del documento no incluyen relación con los marcadores presentados.

Tabla 2. Relación Marcadores y Aspectos/Competencias a Evaluar

\begin{tabular}{|c|c|c|c|c|c|c|c|c|c|c|c|c|c|c|}
\hline & 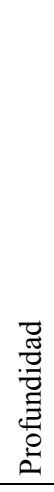 & 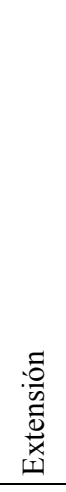 & 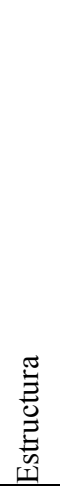 & 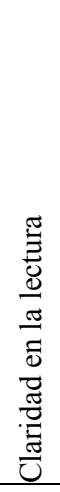 & 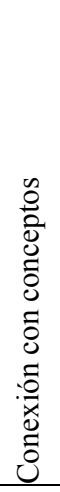 & 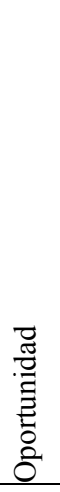 & 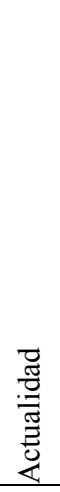 & 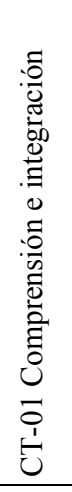 & 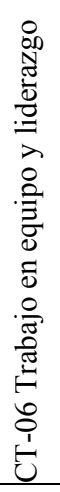 & 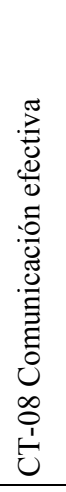 & 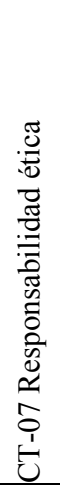 & 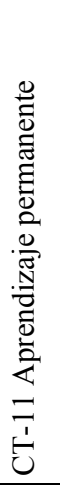 & 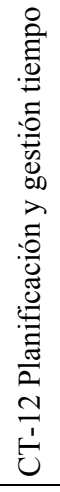 & 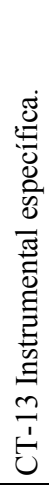 \\
\hline Ortografía y Gramática & & & & $\mathrm{X}$ & & & & & & $\mathrm{X}$ & & & & \\
\hline $\mathrm{N}^{\mathrm{o}}$ de palabras* & $\mathrm{X}$ & $\mathrm{X}$ & & & & & & & & & & $\mathrm{X}$ & & $\mathrm{X}$ \\
\hline $\mathrm{N}^{\mathrm{o}}$ de párrafos* & & $\mathrm{X}$ & & & & & & & & & & $\mathrm{X}$ & & \\
\hline $\mathrm{N}^{\mathrm{o}}$ de frases* & & $\mathrm{X}$ & & & & & & & & & & $\mathrm{X}$ & & \\
\hline $\mathrm{N}^{\mathrm{o}}$ de figuras* & & $\mathrm{X}$ & & & & & & & & & & $\mathrm{X}$ & & $\mathrm{X}$ \\
\hline $\mathrm{N}^{\mathrm{o}}$ de fuentes consultadas* & $\mathrm{X}$ & & & & $\mathrm{X}$ & & $\mathrm{X}$ & & & & $\mathrm{X}$ & $\mathrm{X}$ & & $\mathrm{X}$ \\
\hline $\mathrm{N}^{\mathrm{o}}$ de links externos* & $\mathrm{X}$ & & & & $\mathrm{X}$ & & $\mathrm{X}$ & $\mathrm{X}$ & & & & $\mathrm{X}$ & & $\mathrm{X}$ \\
\hline $\mathrm{N}^{\mathrm{o}}$ de link internos* & $\mathrm{X}$ & & & & $\mathrm{X}$ & & & $\mathrm{X}$ & & & & $\mathrm{X}$ & & $\mathrm{X}$ \\
\hline Conteo de actividad tiempo & & & & & & & & & $X$ & & & $X$ & $X$ & \\
\hline
\end{tabular}

Debe quedar claro que estos marcadores no pretenden ser el criterio único para valorar ni las competencias ni la calidad de la wiki de los alumnos. Pretenden ser una ayuda para la evaluación. Está claro que el criterio del profesor es el que finalmente debe empastar toda esta información proporcionada por los marcadores. Sin embargo, es indiscutible que puede resultar una herramienta de gran ayuda.

\section{Conclusiones}

Es posible encontrar en la literatura experiencias diversas que muestran el uso de las wikis en la docencia universitaria. Sin embargo, el aspecto de la evaluación del trabajo en wiki es una línea de trabajo más emergente. En dicha línea se presenta este trabajo adecuado al actual sistema evaluación universitario español. De acuerdo con el mismo, el profesor debe

(cc) EY-NC-ND 2016, UniversitatPolitècnica de València 
poner una calificación a los alumnos en la asignatura correspondiente (entre 0 y 10) y una calificación a la/s competencia/s transversales (A,B,C y D) que de acuerdo con los planes del título se haya establecido que se han de evaluar en dicha asignatura.

La innovación presentada en este trabajo pretende ayudar al profesor a la realización de dicha tarea de evaluación en el caso de la realización de una actividad wiki. Para ello analiza qué competencias son desarrolladas a través de la wiki, así como qué aspectos debieran valorarse para determinar la calidad de la wiki. El facilitador se presenta al enlazar tanto competencias como aspectos con marcadores automáticos que pueden obtenerse directamente de la wikis por su propia naturaleza de página web.

Como trabajo futuro se plantea incorporar estos marcadores y su relación con competencias y calidad de la wiki en una completa herramienta de medición automática que disponga de un interfaz útil y sencillo para el profesorado.

Agradecimientos: El presente trabajo se ha desarrollado en el marco del PIME 08/15 "WIKI en la docencia: diseño de una herramienta para evaluar la actividad del alumno" de la Universidad Politécnica de Valencia.

\section{Referencias}

ALEXA.Top500.<http://web.archive.org/web/20090302215553/http://www.alexa.com//site//ds//top_s ites?ts_mode $=$ global\&lang=none $>$ [Consulta: 1 de abril de 2016].

ALSINA MAS MITJÀ, J. (Coord.) (2013). Rúbricas para la evaluación de competencias. ICE Universitat de Barcelona y Ediciones Octaedro, Barcelona.

FOUNTAIN R. Wiki pedagogy, Dossiers Technopedagogiques. $<$ http://profetic.org/dossiers/article.php3?id_article=969> [Consulta: 1 de abril de 2016].

FUNDACION WIKIMEDIA. Wikipedia. <https://es.wikipedia.org/> [Consulta: 1 de abril de 2016]

ICE (2015). Proyecto Competencias Transversales UPV. Rúbricas",.Universitat Politècnica de València.

KAY, J., MAISONNEUVE, N., YACEF, K., y REIMANN, P. (2006). "The big five and visualisations of team work activity" en Lecture Notes in Computer Science, vol 4053, p. 197-206.

KOLODNER J., GUZDIAN M. (1996) Effects with and of CSCL: tracking learning in a new paradigm. Mahwah, NJ: Erlbaum.

LAMB A., JOHNSON L. (2007) ."An information skills workout: Wikis and collaborative writing” en Teacher Librarian, vol 71, p. 57-59.

LEUF B., CUNNINGHAM W. (2001). The Wiki Way: Collaboration and Sharing on the Internet, MA: Addison-Wesley.

MOURTOS, N.J. (2003) "Defining, teaching and assessing lifelong learning skills". En 33rd ASEE/IEEE Frontiers in Education Conference, Boulder (Colorado-USA).

ORTEGA, F., GONZALEZ-BARAHONA, J.M., ROBLES, G. (2007). "The top-ten Wikipedias - A

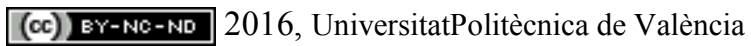

Congreso In-Red (2016) 
quantitative analysis using WikiXRay”. In J. Filipe, B. Shishkov, \& M. Helfert (Eds.), ICSOFT 2007, Proceedings of the Second International Conference on Software and Data Technologies, Volume ISDM/EHST/DC, p. 46-53.

PALOMO-DUARTE, M.; DODERO, J.M.; MEDINA-BULO, I.; RODRÍGUEZ-POSADA, E.J.; RUIZ-RUBE, I. (2014). "Assessment of collaborative learning experiences by graphical analysis of wiki contributions" en Interactive Learning Environments, vol. 22, No. 4, p. 444-466.

PALOMO-DUARTE, M.; RODRÍGUEZ-POSADA, E.J.; MEDINA-BULO, I.; SALES MONTES, N. (2012). "Tecnologías wiki en la docencia de Ingeniería Informática” en ReVisión, vol. 5, No. 1, pp. $1-10$.

PARKER K.R., CHAO J.T. (2007). "Wiki as a Teaching Tool" en Interdisciplinary Journal of Knowledge and Learning Objects, vol 3, p. 1-16.

PUJOLÀS MASET, P. (2009). "La calidad en los equipos de aprendizaje cooperativo: algunas consideraciones para el cálculo del grado de cooperatividad" en Revista de educación, vol. 349.

UNIVERSITAT POLITÈCNICA DE CATALUNYA (2008). Quadern per treballar les competències genèriques a les assignatures. Aprenentatge autònom, Institut de Ciències de l'Educació.

VIÉGAS, F.B., WATTENBERG, M., DAVE, K. (2004). "Studying cooperation and conflict between authors with history flow visualizations". En: SIGCHI conference on Human E., New York, 575-582.

VILLA, A. Y POBLETE, M. (2007). Aprendizaje basado en competencias. Una propuesta para la evaluación de las competencias genéricas. Bilbao, Ediciones Mensajero.

WAGNER, C. (2004). "Wiki: A Technology for Conversational Knowledge Management and Group Collaboration" en Communications of the Association for Information Systems, vol. 13, p. 265.

(cc) EY-NC-ND 2016, UniversitatPolitècnica de València 